\title{
A Robust Protocol for In Vivo THz Skin Measurements
}

\section{Hannah Lindley-Hatcher ${ }^{1}$ - A. I Hernandez-Serrano ${ }^{1}$ • Qiushuo Sun ${ }^{2}$ - Jiarui Wang ${ }^{2}$. Juan Cebrian $^{3} \cdot$ Laurent Blasco $^{4}$ • Emma Pickwell-MacPherson ${ }^{1,2}$ (D)}

Received: 14 June 2019 / Accepted: 14 August 2019 /Published online: 28 August 2019

(C) The Author(s) 2019

\begin{abstract}
This work presents an experimental setup to control the way in which pressure interferes with the repeatability of in vivo $\mathrm{THz}$ skin measurements. By integrating a pressure sensor circuit into our $\mathrm{THz}$ system, it is possible to identify which measurements were taken within a previously specified pressure range. The live response of the pressure sensor helps to acquire data within the desired pressure leading to greater consistency of data between measurements. Additionally, a protocol is proposed to help achieve repeatable results and to remove the effects of the natural variation of the skin through the course of the day. This technique has been shown to be able to quantify the changes induced in the skin following the application of a moisturising skin product and shows the measured result to be significantly different from natural skin variation. This research therefore prepares the way for further studies on the effectiveness of different skin products using in vivo THz measurements.
\end{abstract}

Keywords Terahertz · Moisturisers $\cdot$ Skin $\cdot$ Pressure controlled · Time-domain spectroscopy

\section{Introduction}

Terahertz (THz) imaging is an appealing technology which is increasingly being investigated for potential biomedical applications due to the non-ionising nature and strong sensitivity of $\mathrm{THz}$ light to the water content of biological tissue [1,2]. Potential applications being investigated include the in vivo study of cornea thickness [3], blood glucose levels [4] and the diagnosis of diabetic foot syndrome [5]. THz technology has also attracted interest for its ability to identify cancerous and non-cancerous tissues; this has been demonstrated with many

Emma Pickwell-MacPherson e.macpherson@warwick.ac.uk

1 Department of Physics, University of Warwick, Gibbet Hill Road, Coventry CV4 7AL, UK

2 Department of Electronic Engineering, Chinese University of Hong Kong, Shatin, Hong Kong, China

3 Lipotec SAU, Barcelona, Spain

4 Lubrizol Advanced Materials Inc, Brecksville, OH, USA 
types of cancer including breast [6], colon [7] and oral cancer [8]. However, due to its strong absorption by water, THz light cannot penetrate deeper than the first few hundred micrometres of tissue, and this has led to in vivo skin studies. For example, studies have been conducted which demonstrate the capability of $\mathrm{THz}$ light to identify basal cell carcinomas [9], differentiate dysplastic and non-dysplastic skin nevi [10] and monitor the healing process of scars [11].

It has been shown that many variables need to be controlled if there are to be comparable and consistent results obtained from in vivo THz imaging. For example, Sun et al. demonstrate the effect of occluding the skin on the reflected THz signal [12]. Furthermore, Wang et al. show that variations in the pressure with which the region being imaged contacts the imaging window also changes the THz response of skin [13]. These changes are largely attributed to the changing water distribution within the upper layers of skin due to the skin being unable to breathe and the density of biological matter changing. Due to the high sensitivity of $\mathrm{THz}$ to water content, the changes induced in the skin by the effects, whilst small, can influence the results. These changes to the skin cannot be prevented when the sample must have contact with a quartz imaging window to aid calibration and alignment; therefore, a protocol must be developed so that the effects of occlusion and pressure on the results are consistent [14].

Additionally, due to the sensitivity of $\mathrm{THz}$ light to water content, it can be used to evaluate and/or monitor different skin products which are designed to hydrate the skin. Effective medium theories (EMTs) have already been shown to be capable of retrieving the hydration of the skin as a function of depth from the measured THz reflectivity [15]. The skin can also be modelled with the double Debye model [16], which has been shown to be useful in helping with skin cancer diagnosis [17]. This paper presents the measured THz response of skin before and after the application of a commercially available skin hydrating product. A normalisation of this $\mathrm{THz}$ response is also performed to compensate for the natural variation of the $\mathrm{THz}$ properties of the skin throughout the day.

Companies which produce skin products are interested in quantitatively evaluating the effects of their products using different techniques; however, due to the nature of the research, it is not ethical to apply ionising imaging techniques for in vivo studies. MRI (magnetic resonance imaging) has been used successfully for such purposes [18]; however, these tests are very expensive. One of the techniques presently employed for assessing moisturiser functionality is corneometry which assesses the hydration of the skin indirectly through capacitance measurements [19]; however, it is limited to only measuring down to $20-40 \mu \mathrm{m}$ and is also very sensitive to any changes in conductivity, such as those caused by sweat. Thus, employing $\mathrm{THz}$ techniques to directly determine the hydration of the skin will be a significant improvement [20]. Recently, investigations have been performed using $\mathrm{THz}$ to study the changes induced in the skin resulting from the application of components of skin products [21]. We advance on these studies in this paper by presenting a more robust protocol to control the effects arising from pressure and occlusion to study moisturisers in vivo and a normalisation variable to remove the effects of natural variation of the skin.

This paper therefore proposes a robust and rigorous protocol to obtain repeatable results quantifying the effects of moisturiser products on the skin. Our experimental set up incorporates a pressure sensor device to control the contact pressure of the skin on the imaging window. Additionally, the protocol described in this paper attempts to limit the effect of unknown occlusion times on the measurements by noting the point where initial contact occurs and waiting at least 20 min between measurements to allow the skin to recover from the occlusion effects. We also measure a "control" 
region of skin where we do not apply the product under investigation to account for the natural variation of the skin due to homeostasis and environmental factors.

\section{Method}

\subsection{Experimental Setup}

An electronic circuit capable of converting mechanical pressure into voltage was constructed, as is shown in Fig. 1. A microcontroller board was used to process the measured voltage into a pressure value and turn on the appropriate indicator light. Force-sensitive resistors (FSRs) are connected in a voltage divider circuit using an op-amp to improve stability and repeatability of the output [22]. The output voltage varies with the applied pressure and is inputted into the microcontroller. The FSRs are placed on either side of the imaging window as shown in Fig. 2a and the output from each controls the corresponding indicator LED. If the pressure applied to the FSR is below the previously programmed low-pressure limit, the light will be blue, if the pressure is above the high-pressure limit, the light will be red and finally, if the pressure is within these two limits, the light will be pink. The low-pressure limit chosen for this study was $0.6 \mathrm{~N} \mathrm{~cm}^{-2}$, as this is the lowest value of pressure detectable by the FSRs, and a lower pressure is easier to maintain for a long period of time. The high-pressure limit chosen was $1.1 \mathrm{~N} \mathrm{~cm}^{-2}$, as this allows for some variation in pressure which is below the sensitivity of the $\mathrm{THz}$ system. It is simple to change the desired pressure range depending on the region of skin being measured, the requirements of the experiment and the experience of the subject using the pressure sensor. If the subject is inexperienced, it is beneficial to use a wider pressure range; otherwise, extra errors are introduced as they try to apply the desired pressure; with more experienced volunteers, the desired pressure range can be tighter as this will be

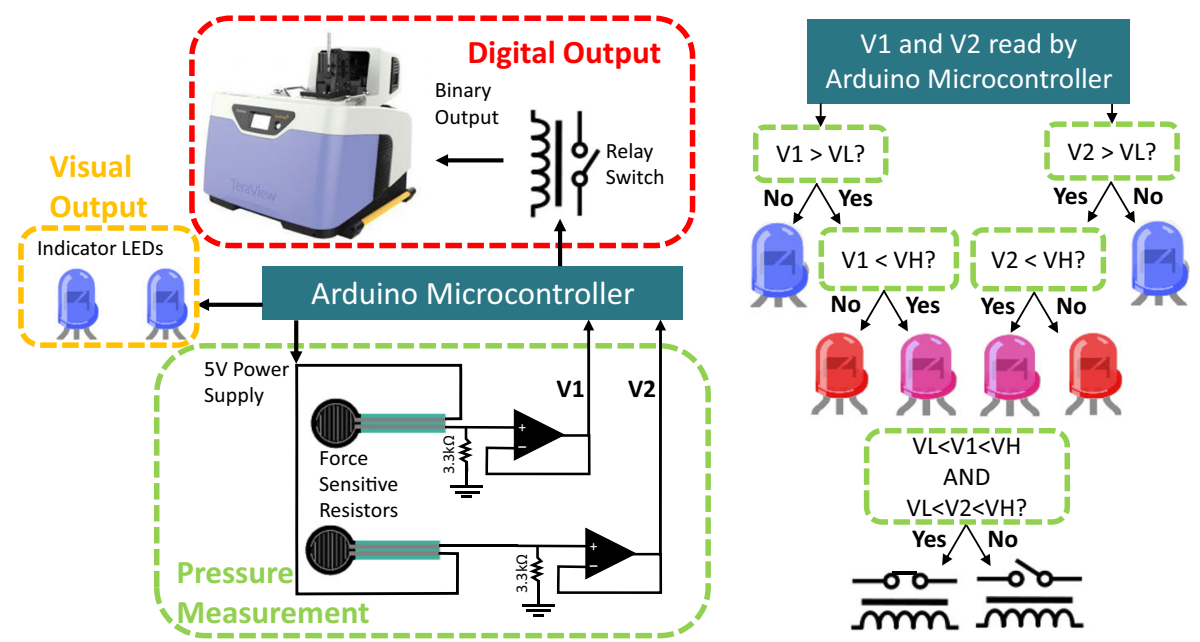

Fig. 1 Diagram of the circuit used to integrate a pressure sensor with the TeraPulse 4000 main unit and a flow chart describing the code used by the Arduino microcontroller to implement this. V1 and V2 are the output voltages from the responses of the two force-sensitive resistors, and VL and VH are the lower and higher threshold voltages for the pressure sensor to respond to respectively. When the voltage is between VL and VH, a pink light is shown to the subject and a binary signal is recorded alongside the THz response to aid processing 


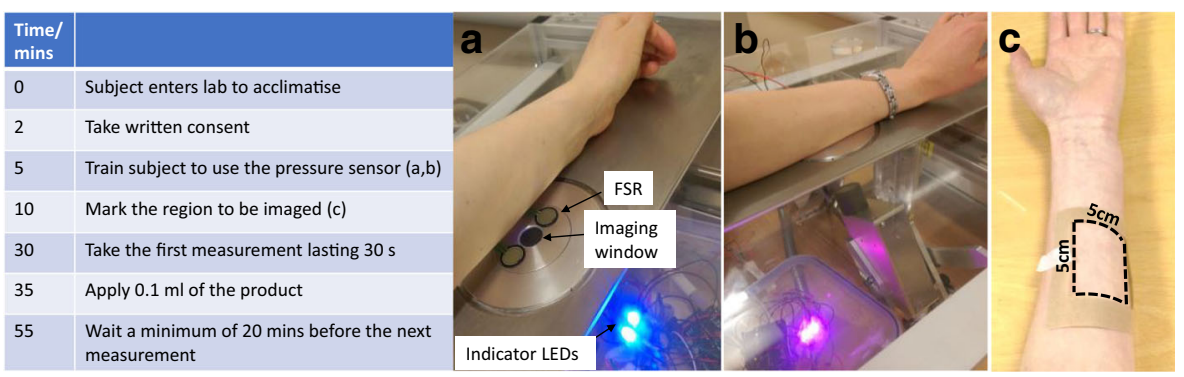

Fig. 2 Table of the protocol used for in vivo skin measurements. a) and b), the experimental setup used with the pressure sensor with applied pressure below the desired limit and within the desired limits respectively (indicated by the blue and pink lights). c) The marked region to be imaged on the volar forearm

achievable without moving, this will significantly reduce the uncertainty introduced by pressure.

Furthermore, the circuit also provides a digital output to the THz system; for this study, the TeraView fibre coupled TeraPulse 4000 spectrometer was used. When the pressure read by the pressure sensor is within the specified range of values, the circuit is closed triggering a signal to the spectrometer whilst recording the $\mathrm{THz}$ signal. After this, the $\mathrm{THz}$ signal is saved in an $\mathrm{h} 5$ file together with a binary mark indicating whether the measurement was recorded when the pressure read by the sensor was in the specified range. This procedure makes it possible to select only the $\mathrm{THz}$ signals recorded in the pre-defined pressure range.

\subsection{Protocol for In Vivo Studies}

A protocol for in vivo skin studies has been designed to allow a more rigorous comparison between measurements taken at different times and from different subjects. The outline of this protocol is displayed in the table in Fig. 2. Before the measurements can be performed, the subject must acclimatise to the controlled temperature and humidity room where the measurements will be taken, with the area to be imaged exposed, e.g. sleeves rolled up. During this adjustment time, the subject is trained to use the pressure sensor system as shown in Fig. 2a and $\mathrm{b}$, to practice applying the correct pressure consistently. The subject is also asked that their arm not be removed from the imaging window at any point during the measurement as this will introduce uncertainty into the occlusion process of the skin. Additionally, the acclimatisation time is used to mark the region to be imaged with a $5 \mathrm{~cm}$ by $5 \mathrm{~cm}$ square $\left(25 \mathrm{~cm}^{2}\right)$ on the volar forearm halfway between the elbow and the wrist, as shown in Fig. 2c). This region was chosen for study as it can be easily placed on the imaging system, also it has few hairs which would interfere with the contact of the skin with the imaging window and has minimal environmental exposure, for example to UV radiation. The marking of the region makes it possible for the same location to be measured repeatedly and helps ensure that the area that the moisturiser product was applied is the area that is imaged. All the experiments were performed with the informed consent of the volunteers.

The imaging parameters which are used result in a 33s measurement consisting of 200 waveforms; this yields measurements at small enough time intervals for dynamic changes in the skin such as occlusion to be observed. To allow the skin to recover from occlusion, we waited 5 min between the first measurement and the application of the skin product. The skin product is measured in 0.1-ml quantities using a Pos-D micropipette from Mettler Toledo; this 
volume is sufficient to allow saturation of the $25 \mathrm{~cm}^{2}$ region with no excess remaining on the skin. This volume can be changed depending on the products being tested and the ability of the skin to absorb them. The product is rubbed into the skin whilst wearing a latex glove to ensure that none of the product is absorbed by the hand applying it, efforts are also made to ensure that the product is massaged into the skin consistently covering all the marked area. We leave an interval of $20 \mathrm{~min}$ between measurements to allow the water distribution in the skin to recover from the effects of occlusion from the first measurement [12].

The product of interest was only applied to the left arm and the right arm remained untreated to act as a control. This control arm will make it possible to identify which observed changes are the result of the moisturiser product and which are induced by natural variation in the skin with time of day, eating, drinking, or other environmental factors. In this study, measurements of the skin were taken before the application of the product and $30 \mathrm{~min}, 1 \mathrm{~h}, 2 \mathrm{~h}$, and $4 \mathrm{~h}$ after the application.

\subsection{Data Processing}

\subsubsection{Calibration and Testing}

The FSRs in the circuit give an output voltage which is a function of the pressure applied. In order to extract a meaningful pressure value from this output, the circuit must be calibrated through the application of weights; the result of this is shown in Fig. 3a. The red and blue lines mark the upper and lower thresholds respectively that the pressure sensor lights respond to and the error bars display the voltage range output during the application of that fixed weight. Figure $3 \mathrm{~b}$ shows the average of 10 consecutive $P$ measurements as a function of weight as determined by the calibrated pressure sensor, where $P$ is the peak-to-peak of the processed signal as defined in Fig. 4c. The $x$ error bars come from the calibration of the pressure sensor and the limit on the range of pressure that the arm can be kept within for sufficient time for the measurement to be taken. The $y$ error bars are the standard deviation of the 10 measurements of $P$. Figure $3 \mathrm{~b}$ shows that for low pressures and sufficiently small pressure ranges the variation in pressure is smaller than present $\mathrm{THz}$ sensitivity. Therefore, using the pressure sensor to limit the variation in pressure to correspond to a weight range of $100 \mathrm{~g}$ is enough to significantly reduce variation due to pressure whilst preventing the subject from moving excessively to attempt to keep within an unattainably small pressure range.

\subsubsection{Processing the Reflected THz Signal}

The $\mathrm{THz}$ response of skin was calculated by processing the measured reflected signals to obtain the impulse function and extract the optical properties of the sample.

$$
\text { Impulse Function }=i F F T\left[\operatorname{FFT}(\text { filter }) \times \frac{\operatorname{FFT}\left(E_{\text {sample }}(t)-E_{\text {baseline }}(t)\right)}{\operatorname{FFT}\left(E_{\text {air }}(t)-E_{\text {baseline }}(t)\right)}\right]
$$

This processed signal is defined in Eq. 1, where $E_{\text {sample }}(t), E_{\text {air }}(t)$, and $E_{\text {baseline }}(t)$ are the measured $\mathrm{THz}$ responses of the sample, air and a baseline where a second quartz window is placed on the imaging window to remove the second reflection. The filter term is a double Gaussian which is used to remove high- and low-frequency noise [12, 23]. 

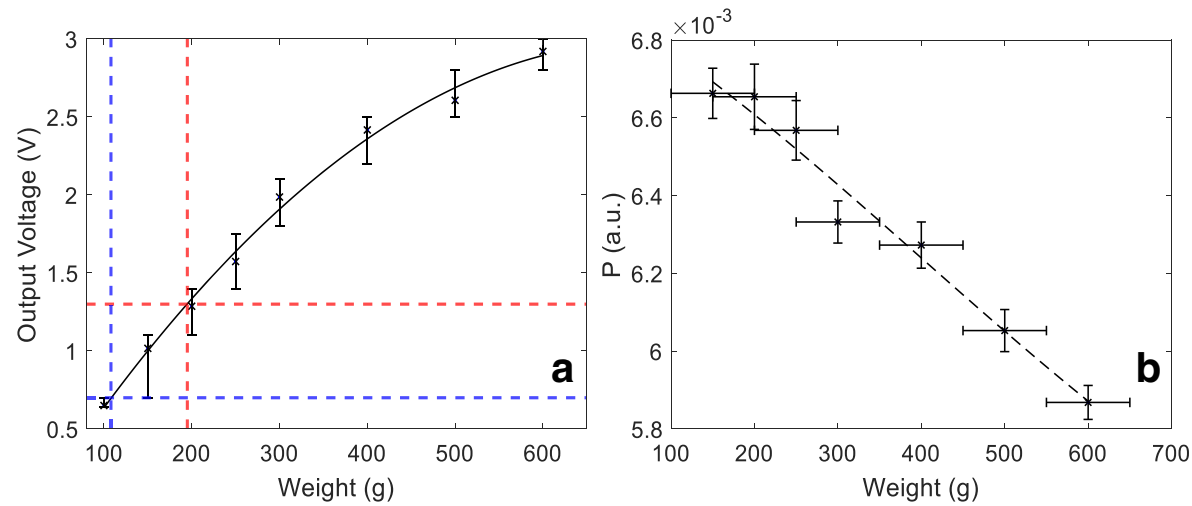

Fig. 3 a) The calibration of the FSRs using known weights and taking an average of 3 readings of the output voltage, the error bars are the variation of the voltage with a fixed weight. The blue and red lines mark the lower and upper voltage and weight thresholds for the blue and red lights to turn on used in this study. b) The measured $P$ at different pressures, the $x$ error bars correspond to the calibration errors in a) and the $y$ errors come from the standard deviation of the 10 measurements which were averaged to give each data point

Sun et al. [4, 9] demonstrated that as the time of occlusion of the skin increases, water builds up in the skin surface and this can be seen as a decrease in $P$, as shown in Fig. 4c. This is because the increased water content in the skin increases the refractive index of skin, so the reflected THz signal is reduced. Therefore, through this initial study into pressure controlled $\mathrm{THz}$ measurements of the effect of applying a moisturiser, a decrease in $P$ is understood to indicate an increase in skin hydration.

\subsubsection{Using the Pressure Sensor Output}

The output from the pressure sensor can be used to identify the point at which the arm makes stable contact with the imaging window and how long the subject took to attain the desired contact pressure. This makes it possible to determine which changes of the $\mathrm{THz}$ pulse are due
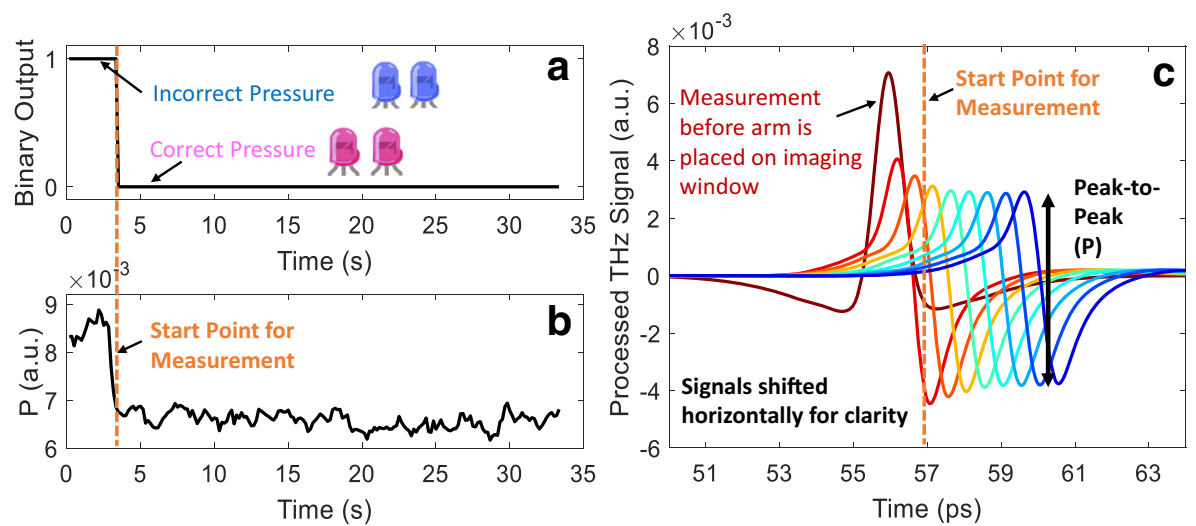

Fig. 4 The output from a THz measurement of skin. The start point of the measurement, where stable contact with the imaging window was reached at the correct pressure, is marked by the orange dashed line. a) The pressure sensor binary output, b) the impulse function Peak-to-Peak $(\mathrm{P}), \mathrm{c})$ the impulse function at the start of the measurement both before and after the contact of the skin with the imaging window. The impulse functions are shifted horizontally for clarity and only one signal of the window before contact is displayed in red 
to occlusion and which are due to an incorrect pressure between the arm and the window. Also, any changes throughout the measurement that are the result of a change in pressure can be identified. If the subject failed to reach the correct pressure quickly or the pressure varies significantly throughout the measurement, the data are excluded.

The processing and analysis of the $\mathrm{THz}$ signals using the pressure sensor data are shown in Fig. 4. The binary output from the pressure sensor as a function of time is displayed in Fig. 4a, and the regions of incorrect pressure are identified. Figure $4 \mathrm{~b}$ shows the variation of $P$ as a function of time with the start point corresponding to the correct pressure marked. Figure $4 \mathrm{c}$ shows the processed $\mathrm{THz}$ response measured at different time intervals into the measurement, including one just before the arm makes contact with the imaging window. These plots are used together to determine the time into the measurement at which the data can be considered useful and consistent with those taken from other subjects. In Fig. 4c, the impulse functions have been shifted horizontally for clarity.

\subsubsection{Normalising Moisturiser Measurements}

To eliminate variation in the skin not due to the moisturiser, we used one arm as control and processed the data with Eq. 2 to yield the normalised relative change (NRC).

$$
\operatorname{NRC}(\%)=100 \times \frac{\left(P_{T t}-P_{T 0}\right)-\left(P_{C t}-P_{C 0}\right)}{P_{T 0}+\left(P_{C t}-P_{C 0}\right)}
$$

Where $P_{T t}$ and $P_{C t}$ are the measured $P$ of the treated and control arms, time $t$ minutes after the application of the product, $P_{T 0}$ and $P_{C 0}$ are the initial $P$ of both arms. NRC represents a percentage change in $P$, resulting from the application of a moisturiser product relative to that of the control arm which should be representative of the natural variation of the arm due to environmental factors. It might be expected that $\left(P_{C t}-P_{C 0}\right)$ would average to zero but when there is a change in the skin not caused by the moisturiser, e.g. due to eating, drinking, or a temperature change, then this will be non-zero and the effect from it will be removed from the change observed on the left arm when the moisturiser was applied. The more negative the NRC value, the more $P$ decreased due to the moisturiser application and therefore the greater the hydration increase in the skin. However, it is also possible that the moisturising product may induce other changes in the skin not linked to hydration which may alter the $\mathrm{THz}$ response, further modelling of the skin is required to identify how much of the observed change in NRC is caused by changes in skin hydration.

The NRC can isolate the change in the THz response of skin induced by the application of the skin product. When the water content of the skin is extracted, a similar normalisation process can be carried out to identify the relative hydration change in the skin.

\section{Results and Discussion}

To test the capabilities of this protocol for quantifying the effects of moisturisers on the skin, a study was conducted using a commercially available skin hydrating product. Using the protocol described in the "Protocol for In Vivo Studies" section. Vaseline lotion was tested on the same subject three times (with 1 week between each study to allow for recovery of the skin where the moisturiser was applied). The results of this study are shown in Fig. 5a, where 
the changes in $P$ of the control and moisturised arm in three repeat measurements on the same subject can be seen. In this figure, the average of 10 consecutive $P$ measurements taken $25 \mathrm{~s}$ into the occlusion process is plotted, and the error bars are the standard deviation of these ten values. The dashed lines indicating the control arm in Fig. 5a are largely self-consistent; however, there are some small variations which can be due to factors other than the application of the moisturiser. If there is an observable change due to the consumption of food or other factors which influence the skin homogeneously on the left and right arms, the use of the NRC variable should remove this effect. Additionally, a large change can be seen between measurements 1, 2 and 3 of the control arms, using the NRC variable will yield a measure of the change of the state of the skin relative to the starting conditions before the application of the product. There is a significant decrease in $P$ shown by the solid lines after the application of the Vaseline, this change is large enough that it cannot be accounted for simply by natural variation of the skin; therefore, this figure demonstrates that $\mathrm{THz}$ is capable of characterising changes in the skin induced by the application of skin products.

Figure $5 \mathrm{~b}$ shows the result of three identical studies on the same subject with the treated arm normalised to the control arm; the data point plotted for the value of the NRC before the application of the moisturising product is defined to be zero by Eq. 2 and therefore has no associated error. A significant change is observed in the skin following the application of the product as none of the results overlap with dashed line marking out zero NRC. There is a consistent and significant decrease in the NRC of approximately 10-15\%, following the application of the product. As all of the normalised results are significantly different from zero, this change cannot be attributed to the natural variation of the skin. The pressure sensor was used to identify the start point of the occlusion of the skin which was then used to align the start points of the data from the control and treated regions. By doing this before calculating the NRC, the effect of occlusion is compensated for so this should not contribute to the error bars which are the standard deviation of the 10 consecutive NRC values averaged to give the values in the figure. This figure suggests that after the initial changes observed when the product is applied, further increases in this change can be seen at increasing time after the
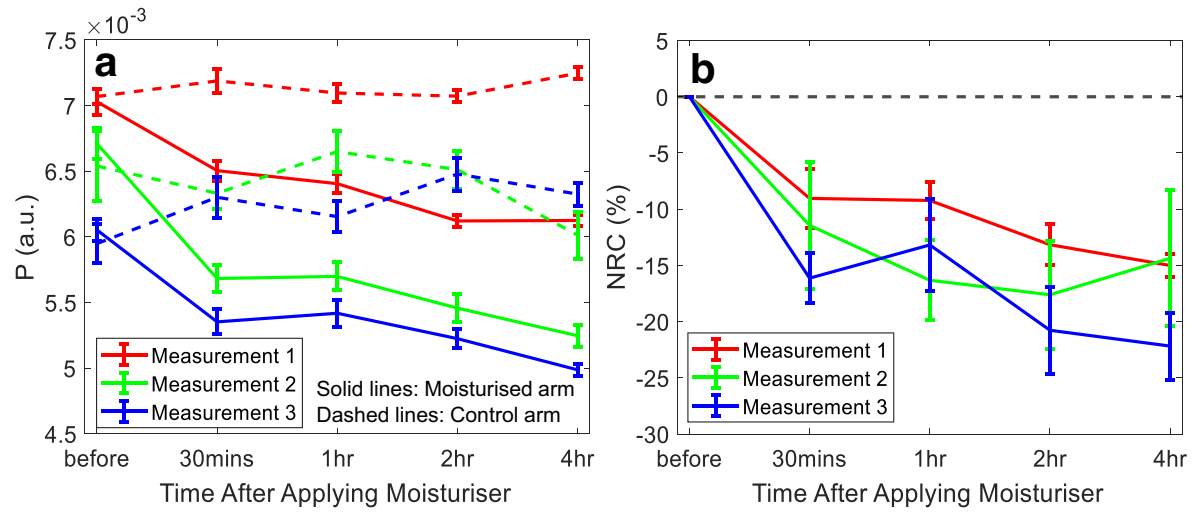

Fig. 5 The THz response of skin after the application of a commercial skin product. a) The average of 10 measurements of $P$ plotted at different time intervals before and after applying moisturiser to one arm. b) The NRC observed after $25 \mathrm{~s}$ of occlusion in three repeats of the same protocol on the same subject after the application of the product, the data point marked for the NRC value before the application is defined to be zero by Eq. 2 and the dashed black line highlights the point for no normalised change observed. In both figures, the error bars correspond to the standard deviation 
application, suggesting that the changes the product induces in the skin may take some time to begin.

This protocol is shown to be capable of producing repeatable in vivo THz skin measurements as the results in Fig. 5b of the percentage NRC of the skin largely agree within their error bars despite the measurements being taken at 1-week intervals. From these results, the NRC of the skin 30 min after the application of the skin product is shown to decrease to $10 \%$ and $4 \mathrm{~h}$ after the application of the product, this decreases further to $17 \%$. However, further measurements will need to be taken on a range of subjects before we can be confident that this trend applies to all skin types in a range of seasons.

This work can be expanded on in the future by applying the protocol to a wider range of subjects and samples to study if a change in the effects can be observed. A model could also be developed to facilitate the extraction of the hydration as a function of depth to allow the observation of the effects of the products deeper into the skin. This would also give greater meaning to the effect of applying the products if they can be expressed in terms of percentage increase in hydration, which will be of more interest to potential skin product customers.

\section{Conclusion}

In summary, a pressure sensor circuit was constructed to give a real-time and digital output indicating whether the contact pressure of the arm on the imaging window is within the desired range. Using this experimental setup to measure all the parameters needed to calculate the $\mathrm{NRC}$, the change in the $\mathrm{THz}$ response of skin after the application of a commercial skin product was shown to be significant and beyond what can be attributed to natural variation of the skin. This paper therefore presents a robust protocol for in vivo THz skin measurements which we envision will serve as a valuable guide to those conducting $\mathrm{THz}$ in vivo skin measurements in the future.

Funding information This work was partially supported by the Research Grants Council of Hong Kong (project number 14206717), The EPSRC (project number EP/S021442/1) the Royal Society Wolfson Merit Award and the Hong Kong PhD Fellowship Award.

\section{Compliance with Ethical Standards}

Conflict of Interest The authors declare that they have no conflict of interest.

Open Access This article is distributed under the terms of the Creative Commons Attribution 4.0 International License (http://creativecommons.org/licenses/by/4.0/), which permits unrestricted use, distribution, and reproduction in any medium, provided you give appropriate credit to the original author(s) and the source, provide a link to the Creative Commons license, and indicate if changes were made.

\section{References}

1. Q. Sun, Y. He, K. Liu, S. Fan, E. P. J. Parrott, and E. Pickwell-MacPherson, Recent advances in terahertz technology for biomedical applications, Quant. Imaging Med. Surg., vol. 7, no. 3, pp. 345-355, 2017.

2. E. Pickwell and V. P. Wallace, Biomedical applications of terahertz technology, J. Phys. D. Appl. Phys., vol. 39 , no. 17,2006 
3. Z. D. Taylor et al., THz and mm-wave sensing of corneal tissue water content: In vivo sensing and imaging results, IEEE Trans. Terahertz Sci. Technol., 2015.

4. O. Cherkasova, M. Nazarov, and A. Shkurinov, Noninvasive blood glucose monitoring in the terahertz frequency range, Opt. Quantum Electron., vol. 48, no. 3, pp. 1-12, 2016.

5. G. G. Hernandez-Cardoso et al., Terahertz imaging for early screening of diabetic foot syndrome: A proof of concept, Sci. Rep., vol. 7, no. 42124, 2017.

6. A. J. Fitzgerald et al., Terahertz Pulsed Imaging of Human Breast Tumors, Radiology, vol. 239, no. 2, pp. 533-540, 2006.

7. F. Wahaia et al., Terahertz absorption and reflection imaging of carcinoma-affected colon tissues embedded in paraffin, J. Mol. Struct., vol. 1107, pp. 214-219, 2016.

8. Y. C. Sim, J. Y. Park, K.-M. Ahn, C. Park, and J.-H. Son, Terahertz imaging of excised oral cancer at frozen temperature, Biomed. Opt. Express, vol. 4, no. 8, 2013.

9. V. P. Wallace et al., Terahertz pulsed imaging of basal cell carcinoma ex vivo and in vivo, Br. J. Dermatol., vol. 151, no. 2, pp. $424-432,2004$.

10. K. I. Zaytsev et al., In vivo terahertz pulsed spectroscopy of dysplastic and non-dysplastic skin nevi, J Phys Conf Ser, vol. 735, no. 12076.

11. S. Fan, B. S. Y. Ung, E. P. J. Parrott, V. P. Wallace, and E. Pickwell-Macpherson, In vivo terahertz reflection imaging of human scars during and after the healing process, J. Biophotonics, vol. 10, pp. 1143-1151, 2017.

12. Q. Sun, E. P. J. Parrott, Y. He, and E. Pickwell-MacPherson, In vivo THz imaging of human skin: Accounting for occlusion effects, J. Biophotonics, vol. 11, no. 2, pp. 1-8, 2018.

13. J. Wang, R. I. Stantchev, Q. Sun, T.-W. Chiu, A. T. Ahuja, and E. Pickwell-Macpherson, THz in vivo measurements : the effects of pressure on skin reflectivity, Biomed. Opt. Express, vol. 9, no. 12, pp. 64676476, 2018.

14. S. Fan, E. P. J. Parrott, B. S. Y. Ung, and E. Pickwell-MacPherson, Calibration method to improve the accuracy of THz imaging and spectroscopy in reflection geometry, Photonics Res., vol. 4, no. 3, p. A29, 2016.

15. D. B. Bennett, W. Li, Z. D. Taylor, W. S. Grundfest, and E. R. Brown, Stratified media model for Terahertz reflectometry of the skin, IEEE Sens. J., vol. 11, no. 5, pp. 1253-1262, 2011.

16. B. C. Q. Truong, H. D. Tuan, H. H. Kha, and H. T. Nguyen, Debye parameter extraction for characterizing interaction of terahertz radiation with human skin tissue, IEEE Trans. Biomed. Eng., 2013.

17. B. C. Q. Truong, H. D. Tuan, A. J. Fitzgerald, V. P. Wallace, and H. T. Nguyen, High correlation of double Debye model parameters in skin cancer detection, in 2014 36th Annual International Conference of the IEEE Engineering in Medicine and Biology Society, EMBC 2014, 2014.

18. E. Ciampi et al., Dynamic in vivo mapping of model moisturiser ingress into human skin by GARfield MRI, NMR Biomed., vol. 24, no. 2, pp. 135-144, 2011.

19. A. L. Vergnanini, V. Aoki, R. Takaoka, and J. Madi, Comparative effects of pimecrolimus cream vehicle and three commercially available moisturizers on skin hydration and transepidermal water loss, J. Dermatolog. Treat., vol. 21, pp. 126-129, 2010.

20. Q. Sun et al., In vivo estimation of water diffusivity in occluded human skin using terahertz reflection spectroscopy, J. Biophotonics, p. e201800145, 2018.

21. M. Alfaro, L. H. Martinez-Meza, C. Rojas-Landeros, and E. Castro-Camus, Evaluation of skin moisturizer effects using terahertz time domain imaging, Photonics Dermatology Plast. Surg. 2018, no. February, p. 26, 2018.

22. Interlink Electronics, Force Sensing Resistor Integration Guide and Evaluation Parts Catalog. 2018.

23. X. Chen, E. P. J. Parrott, B. S. Y. Ung, and E. Pickwell-Macpherson, A Robust Baseline and Reference Modification and Acquisition Algorithm for Accurate THz Imaging, IEEE Trans. Terahertz Sci. Technol., vol. 7, no. 5, pp. 493-501, 2017.

Publisher's Note Springer Nature remains neutral with regard to jurisdictional claims in published maps and institutional affiliations. 